Reprinted from:

Algebraic Transformation Groups and Algebraic Varieties, Encyclopaedia of Mathematical Sciences, Vol. 132,

Subseries Invariant Theory and Algebraic Transformation Groups, Vol. III, Springer-Verlag, 2004

\title{
THE ROLE OF EXOTIC AFFINE SPACES IN THE CLASSIFICATION OF HOMOGENEOUS AFFINE VARIETIES
}

\author{
DENNIS SNOW
}




\title{
The Role Of Exotic Affine Spaces In the Classification Of Homogeneous Affine Varieties
}

\author{
Dennis Snow* \\ Department of Mathematics, University of Notre Dame, Notre Dame, IN \\ 46556-4618, USA. snow.1@nd.edu
}

Let $G$ be a connected linear algebraic group over $\mathbb{C}$ and let $H$ a closed algebraic subgroup. A fundamental problem in the study of homogeneous spaces is to describe, characterize, or classify those quotients $G / H$ that are affine varieties. While cohomological characterizations of affine $G / H$ are possible, there is still no general group-theoretic conditions that imply $G / H$ is affine. In this article, we survey some of the known results about this problem and suggest a way of classifying affine $G / H$ by means of its internal geometric structure as a fiber bundle.

Cohomological characterizations of affine $G / H$ provide useful vanishing theorems and related information if one already knows $G / H$ is affine. Such characterizations cannot be realistically applied to prove that a given homogeneous space $G / H$ is affine. Ideally, one would like to have easily verified group-theoretic conditions on $G$ and $H$ that imply $G / H$ is affine. Very few positive results are known in this direction, the most notable of which is Matsushima's Theorem for reductive groups. For general linear algebraic groups there is a natural generalization of Matsushima's Theorem that provides a necessary condition for $G / H$ to be affine. While this criterion is also sufficient for some special situations, it is not sufficient in general.

In the absence of general group-theoretic conditions for $G / H$ to be affine, it is worthwhile to understand the underlying geometric structure of an affine homogeneous space $G / H$. Such a space is always isomorphic to a fiber bundle over an orbit of a maximal reductive subgroup of $G$. The fiber is a smooth affine variety diffeomorphic to an affine space $\mathbb{C}^{n}$. Here several interesting phenomena seem possible: either the fiber is truly an "exotic" affine space or is in fact isomorphic to $\mathbb{C}^{n}$. If exotic structures occur, they would also provide counter-examples to the Cancellation Problem for affine spaces. So far, no such exotic examples are known. If such structures are impossible, then an affine homogeneous space $G / H$ would always have the simple description of

\footnotetext{
* Supported by the Erwin Schrödinger Institute, Vienna, Austria
} 
a homogeneous vector bundle. In this case, one can change groups, $G / H=$ $\hat{G} / \hat{H}$, where $\hat{G}$ and $\hat{H}$ are easily classified, giving an indirect group-theoretic characterization for $G / H$ to be affine.

\section{Cohomological Characterizations}

Recall that a subgroup $H \subset G$ is called observable if every finite dimensional rational $H$-module can be embedded as an $H$-submodule of a finite dimensional rational $G$-module. This is equivalent to the condition that for any rational $H$-module $V$, the induced module $\left.V\right|^{G}=\left\{s: G \rightarrow V \mid s\left(g h^{-1}\right)=\right.$ $h \cdot s(g), \forall h \in H, \forall g \in G\}$ surjects onto $V$ under the evaluation map $s \rightarrow s(1)$. It is well-known that $H$ is observable in $G$ if and only if $G / H$ is quasi-affine [2]. The subgroup $H$ is call strongly observable if, given any rational $H$ module $V$, $V$ is an $H$-submodule of a rational $G$-module $W$ such that $V^{H}=W^{G}$. Finally, $H$ is called an exact subgroup of $G$ if induction from rational $H$-modules to rational $G$-modules preserves short exact sequences.

Theorem 1. [4, 13] The following are equivalent:

1. $G / H$ is affine.

2. $H$ is a strongly observable subgroup of $G$.

3. $H$ is an exact subgroup of $G$.

4. $H^{1}\left(R_{u}(H), \mathcal{O}(G)\right)=0$ (or, equivalently, $H^{1}\left(G / R_{u}(H), \mathcal{O}\right)=0$ ) where $R_{u}(H)$ is the unipotent radical of $H$.

Such characterizations of affineness are basically "cohomological" in nature. They are primarily used when one already knows that $G / H$ is affine. Verifying the properties themselves may be more difficult than directly proving that $G / H$ is affine.

\section{Group-theoretic Conditions}

There is a practical need for easily verified conditions on the groups $G$ and $H$ that guarantee the quotient $G / H$ is affine. We shall now investigate some of the known results in this direction.

\subsection{Unipotent and Solvable Groups}

If $G$ is a unipotent linear algebraic group, then $G / H \cong \mathbb{C}^{n}$ for any algebraic subgroup $H$. More generally, if $G$ is a solvable linear algebraic group, then $G / H \cong \mathbb{C}^{n} \times\left(\mathbb{C}^{*}\right)^{m}$. The corresponding statements for complex Lie groups are not automatically true. For example if $G=\mathbb{C}^{*} \times \mathbb{C}^{*}$ and $H=\left\{\left(e^{z}, e^{i z}\right) \mid z \in\right.$ $\mathbb{C}\}$, then $G / H$ is a compact complex torus. Nevertheless, some generalizations are possible, see [13]. 


\subsection{Reductive Groups}

After these relatively simple cases, the best known group-theoretic criterion for $G / H$ to be affine goes back to Matsushima [8]:

If $G$ is reductive then $G / H$ is affine if and only if $H$ is reductive.

Matsushima's original theorem assumes $G$ is a reductive complex Lie group and characterizes when $G / H$ is Stein. However, a reductive complex Lie group $G$ is in fact biholomorphically isomorphic to an algebraic group [6] and $G / H$ is affine if it is Stein [1]. Matsushima's theorem has been generalized to reductive algebraic groups over algebraically closed fields of positive characteristic, see $[11,3]$.

\subsection{General Linear Algebraic Groups}

Any connected linear algebraic group $G$ has a decomposition into a semi-direct product, $G=M \cdot R_{u}(G)$, where $M$ is a maximal reductive subgroup $M$ and $R_{u}(G)$ is the unipotent radical of $G$. A closed algebraic subgroup $H$ has a similar decomposition, $H=L \cdot R_{u}(H)$ where $L$ is a maximal reductive subgroup of $H$ (not necessarily connected). Since the maximal reductive subgroups of $G$ are conjugate, we may assume $L \subset M$. The group $L$ is not important in determining whether $G / H$ is affine:

$G / H$ is affine if and only if $G / R_{u}(H)$ is affine.

This follows from the fact that $L$ is reductive and $G / R_{u}(H) \rightarrow G / H$ is a principal $L$-bundle, see [11]. We therefore focus our attention on $R_{u}(H)$ and its location in $G$.

If $R_{u}(H) \subset R_{u}(G)$, then, of course, $G / R_{u}(H) \cong M \times R_{u}(G) / R_{u}(H)$ is affine and so $G / H$ is affine. However, $R_{u}(H) \subset R_{u}(G)$ is not a necessary condition for $G / H$ to be affine. For example, let $G$ be the semi-direct product $\operatorname{SL}(2, \mathbb{C}) \cdot U$ where $U$ is the standard 2-dimensional representation of $\mathrm{SL}(2, \mathbb{C})$, and let

$$
H=\left\{\left[\begin{array}{ll}
1 & 0 \\
t & 1
\end{array}\right] \times(0, t) \mid t \in \mathbb{C}\right\} .
$$

Then $G / H \cong \operatorname{SL}(2, \mathbb{C}) \times \mathbb{C}$.

A necessary condition for $G / H$ to be affine is not hard to discover, see $[4,13]$.

Lemma 1. If $G / H$ is affine then the intersection of $R_{u}(H)$ with any reductive subgroup of $G$ is trivial.

Proof. Let $M$ be a maximal reductive subgroup of $G$. We must show that $R_{u}(H) \cap M^{g}=1$ for all $g \in G$. Since $G / H$ affine, so is $G / R_{u}(H)$, and thus any $M$-orbit of minimal dimension in $G / R_{u}(H)$, being automatically closed, is affine, see [10]. By Matsushima's Theorem, the isotropy subgroup in $M$ of 
such an orbit is reductive. However, it also is of the form $M \cap R_{u}(H)^{g}$ for some $g \in G$, and, being a subgroup of a the unipotent algebraic group $R_{u}(H)^{g}$, is unipotent. Therefore, $M \cap R_{u}(H)^{g}=1$ and any $M$-orbit of minimal dimension is isomorphic to $M$. Hence, all $M$-orbits have the same dimension. Therefore every $M$ orbit in $G / G_{u}(H)$ is isomorphic to $M$ and $M \cap R_{u}(H)^{g}=1$ for all $g \in G$.

The above lemma also holds when $G / H$ is Stein [13]. Notice that the lemma is a natural generalization of Matsushima's Theorem in one direction: if $G$ is reductive and $G / H$ is affine, then the lemma implies $R_{u}(H)=1$ and $H$ is reductive. It is natural to explore whether the converse to Lemma 1 holds. For convenience, let us say that $H$ satisfies Matsushima's criterion in $G$ if the intersection of $R_{u}(H)$ with any reductive subgroup of $G$ is trivial, or equivalently, if $R_{u}(H) \cap M^{g}=1$ for all $g \in G$.

If $H$ satisfies Matsushima's Criterion, then, as we have seen in the proof of the lemma, a maximal reductive subgroup $M$ of $G$ acts freely on $G / R_{u}(H)$. It is easy to see that this condition is equivalent to $R_{u}(H)$ acting freely on $M \backslash G$ from the right. Since $M \backslash G \cong R_{u}(G) \cong \mathbb{C}^{n}$, we are led to considering free actions of unipotent groups on affine space. In fact, in this situation, the actions are also triangular [14]. However, free unipotent triangular actions on $\mathbb{C}^{n}$ can be badly behaved in general: the quotient may not be separated and may not be affine even if it is separated [15]. Nevertheless, this point of view does yield positive results in certain simple cases.

Theorem 2. $[4,14]$ Let $G$ be a linear algebraic group and let $H$ be a closed subgroup. Assume that $\operatorname{dim} R_{u}(H) \leq 1$ or $\operatorname{dim} R_{u}(G) \leq 3$. Then $G / H$ is affine if and only if $H$ satisfies Matsushima's criterion.

Matsushima's Criterion is not sufficient in general. The following example was discovered by Winklemann [15]: Let $M=\mathrm{SL}(6, \mathbb{C})$ and let

$$
G=\left\{\left[\begin{array}{ll}
1 & 0 \\
z & A
\end{array}\right] \mid z \in \mathbb{C}^{6}, A \in M\right\} \cong M \times \mathbb{C}^{6} .
$$

Let

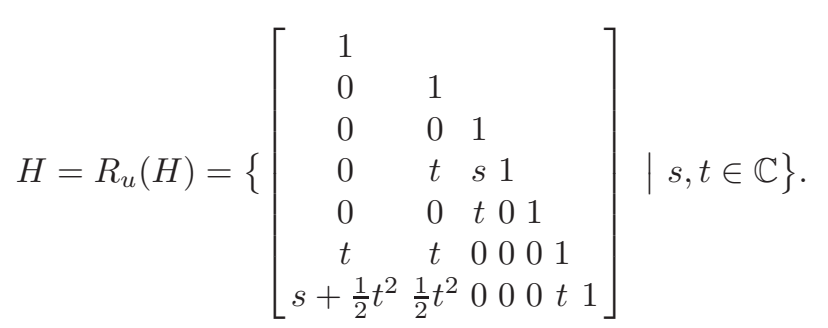

It is not hard to verify that $H$ satisfies Matsushima's Criterion, and that the quotient $M \backslash G / H$ exists and is a smooth contractible quasi-affine variety. However, direct calculation with invariants shows that $X=G / H$ is not affine. In fact, $M \backslash G / H$ is a smooth four-dimensional affine quadric with a 2-codimensional subspace removed. 


\section{Geometric Description}

We now take a closer look at the underlying geometry of an affine homogeneous space $G / H$. As before, we let $M$ be a maximal reductive subgroup of $G$ and let $L \subset M$ be a maximal reductive subgroup of $H$. Let $U=R_{u}(G)$ and $V=R_{u}(H)$ be the unipotent radicals of $G$ and $H$, respectively. If $G / H$ is affine, then we know by Lemma 1 that $M$ acts freely on $G / V$ and, equivalently, that $V$ acts freely on $M \backslash G=U$. Since $G / V$ is affine and $M$ is a reductive group acting freely, Hilbert's theorem on invariants implies that the geometric quotient $M \backslash G / V$ is affine. In fact, the local description of $G / V \rightarrow Y$ in terms of "slices," shows that the quotient map is a locally trivial principal bundle with fiber $M$, see $[7,12]$. Consider the following diagram

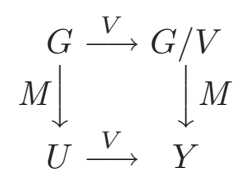

where the vertical maps are the quotients by $M$ and the horizontal maps are the quotients by $V$. Using local sections of $G \rightarrow Y$, we see that the fibration $U \rightarrow Y$ is also a locally trivial principal $V$-bundle. Thus, $Y$ is the base of a locally trivial fibration where both the total space $U \cong \mathbb{C}^{n}$ and the fiber $V \cong \mathbb{C}^{m}$ are affine spaces.

Proposition 1. If $G / H$ is affine, then $G / V$ is $M$-equivariantly isomorphic to $M \times Y$ and $U$ is $V$-equivariantly isomorphic to $Y \times V$.

Proof. The principal $V$-bundle $U \rightarrow Y$ is topologically trivial because the structure group is contractible, and this immediately implies that the bundle is holomorphically trivial [5]. To see that it is also algebraically trivial, we proceed by induction on $\operatorname{dim} V$. If $\operatorname{dim} V=1$, then the triangular action of $V$ on $U$ is equivalent to a translation, see $[4,14]$ which implies $U \cong Y \times V$. If $\operatorname{dim} V>1$, then there is a normal subgroup $V_{1} \subset V$ such that $\operatorname{dim} V / V_{1}=1$. The bundle $G / V_{1} \rightarrow G / V$ with fiber $V / V_{1} \cong \mathbb{C}$ is trivial because $H^{1}(G / V, \mathcal{O})=0$. In particular, $G / V_{1}$ is affine, and hence the quotient $Y_{1}=M \backslash G / V_{1}$ exists and is affine. Since $H^{1}(Y, \mathcal{O})=0$, the principal $V / V_{1}$-bundle $Y_{1} \rightarrow Y$ is also trivial. By induction, $U \cong Y_{1} \times V_{1}$, and therefore, $U \cong Y \times V / V_{1} \times V_{1} \cong Y \times V$. Finally, composing a global section $Y \rightarrow U$ with the projection $G \rightarrow G / V$ gives a global section $Y \rightarrow G / V$, and this implies $G / V$ is $M$-equivariantly isomorphic to $M \times Y$.

\subsection{Cancellation Problem}

The Cancellation Problem is the following: if $\mathbb{C}^{n} \cong Y \times \mathbb{C}^{m}$, is $Y \cong \mathbb{C}^{n-m}$ ? Since $U \cong \mathbb{C}^{n}$ and $V \cong \mathbb{C}^{m}$, the isomorphism $U \cong Y \times V$ of Proposition 1 
gives an example of the Cancellation Problem. This problem remains unsolved in general, but has a positive answer if $\operatorname{dim} Y \leq 2[9]$.

Obviously, $Y$ is a smooth contractible affine variety. If $\operatorname{dim} Y \geq 3$, then $Y$ is in fact diffeomorphic to $\mathbb{C}^{n-m}$, [16]. If $Y$ is not algebraically isomorphic to $\mathbb{C}^{n-m}$ then $Y$ is called an exotic affine space. Exotic affine spaces are known to exist, although no examples are known in the context of the Cancellation Problem, [16]. The relative simplicity of the subgroup $H$ in (2) leads one to believe that it may indeed be possible to create exotic affine spaces of the form $Y=M \backslash G / V=U / V$ which would provide a negative answer to the Cancellation Problem at the same time.

\subsection{Homogeneous Bundle Structure}

The isomorphisms of Proposition 1 provide a natural bundle structure on an affine homogeneous space $G / H$.

Theorem 3. Let $L$ be a maximal reductive subgroup of $H$ and let $M$ be a maximal reductive subgroup of $G$ containing $L$. If $X=G / H$ is affine then $X$ is isomorphic to a homogeneous bundle over $M / L, X \cong M \times_{L} Y \rightarrow M / L$, with fiber $Y$ a smooth contractible affine variety.

Proof. The reductive group $L$ acts by conjugation on both $U=R_{u}(G)$ and $V=R_{u}(H)$ and these actions are isomorphic to a linear representations. By Proposition 1, the $V$-equivariant isomorphism $U \cong Y \times V$ yields a $V$ equivariant map $s: U \rightarrow V$ satisfying $s(u v)=s(u) v$ for all $u \in U, v \in V$.

If we average $s$ over a maximal compact subgroup $K$ of $L$,

$$
\hat{s}(u)=\int_{k \in K} k^{-1} s\left(k u k^{-1}\right) k d k, \quad u \in U
$$

(where $d k$ is some invariant measure on $K$ ), then $\hat{s}$ is still $V$-equivariant. Moreover, since $K$ is Zariski-dense in $L, \hat{s}\left(l u l^{-1}\right)=l \hat{s}(u) l^{-1}$ for all $l \in L, u \in U$. If we identify $Y$ with the $L$-invariant subvariety $\hat{s}^{-1}(1) \subset U$, we obtain a natural action of $L$ on $Y$ and the isomorphism $U \cong Y \times V$ is $L$-equivariant. Moreover, the right $L$ action on $G / V$ preserves the decomposition of Proposition $1, G / V \cong M \times Y$, so that $G / H$ is isomorphic to the homogeneous bundle $M \times{ }_{L} Y=M \times Y / \sim$ where $(m, y) \sim\left(m l^{-1}, l \cdot y\right)$, for all $m \in M, y \in Y$, $l \in L$.

If the homogeneous bundle of Theorem 3 is a homogeneous vector bundle, then it is possible to "change" the groups $G$ and $H$ so that their maximal reductive subgroups and unipotent radicals are aligned.

Theorem 4. Let $X=G / H$ be affine and let $X=M \times{ }_{L} Y \rightarrow M / L$ be the homogeneous bundle of Theorem 3. If $Y$ is isomorphic to a linear representation of $L$, then there exist linear algebraic groups $\hat{G}=M \cdot R_{u}(\hat{G})$ and $\hat{H}=L \cdot R_{u}(\hat{H})$ such that $G / H \cong \hat{G} / \hat{H}$ and $R_{u}(\hat{H}) \subset R_{u}(\hat{G})$. 
Proof. By Theorem 1, the sections $\left.H^{0}(M / L, X) \cong Y\right|^{M}$ generate the bundle. Therefore, there exists a finite dimensional $M$-submodule $U \subset H^{0}(M / L, X)$ that spans the vector space fiber $Y$ over the identity coset $z_{0} \in M / L$. The semi-direct product $\hat{G}=M \cdot U$ then acts on $X=M \times{ }_{L} Y$ by $(m, u) \cdot\left[m^{\prime}, y\right]=$ $\left[m m^{\prime}, y+u\left(m^{\prime}\right)\right]$ for all $m, m^{\prime} \in M, y \in Y$, and $u \in U$. (Recall that the section $u$ is an $L$-equivariant map $u: M \rightarrow Y, u\left(m l^{-1}\right)=l u(m)$ for all $m \in M, l \in L$.) This action is clearly transitive, because the sections $U$ span $Y$ over the identity coset $z_{0} \in M / L$. The isotropy subgroup of the point $[1,0] \in M \times{ }_{L} Y$ is easily computed to be the semi-direct product $\hat{H}=L \cdot V$ where $V=\{u \in U \mid u(L)=0\}$.

\section{References}

1. Barth, W., Otte, M.: Invariante holomorphe Functionen auf reduktiven Liegruppen. Math. Ann. 201 97-112 (1973)

2. Białynicki-Birula, A., Hochschild, G. Mostow, G.D.: Extensions of representations of algebraic linear groups. Am. J. Math. 85 131-144 (1963)

3. Borel, A.: On affine algebraic homogeneous spaces. Arch. Math. 45 74-78 (1985)

4. Cline, E., Parshall B., Scott L.: Induced modules and affine quotients. Math. Ann. 230 1-14 (1977)

5. Grauert, H.: Analytische Faserungen über holomorph-vollständingen Räumen. Math. Ann. 135 263-273 (1958)

6. Hochschild, G. Mostow, G.: Representations and representative functions of Lie groups, III. Ann. Math. 70 85-100 (1959)

7. Luna, D.: Slices étales. Bull. Soc. Math. France, Mémoire 33 81-105 (1973)

8. Matsushima, Y.: Espaces homogènes de Stein des groupes de Lie complexes. Nagoya Math. J. 18 153-164 (1961)

9. Miyanishi, M., Sugie, T.: Affine surfaces containing cylinderlike open sets. J. Math. Kyoto Univ. 20 11-42 (1980)

10. Mumford, D., Fogarty, J.: Geometric Invariant Theory, 2nd edn. (Springer, Berlin, Heidelberg, New York 1982)

11. Richardson, R.: Affine coset spaces of affine algebraic groups. Bull. London. Math. Soc. 91 38-41 (1977)

12. Snow, D.M.: Reductive group actions on Stein spaces. Math. Ann. 259 79-97 (1982)

13. Snow, D.M.: Stein quotients of connected complex Lie groups. Manuscripta Math. 50 185-214 (1985)

14. Snow, D.M.: Triangular actions on $\mathbf{C}^{3}$. Manuscripta Math. 60 407-415 (1988)

15. Winkelmann, J.: On Stein homogeneous manifolds and free holomorphic $\mathbf{C}$ actions on $\mathbf{C}^{n}$. Math. Ann. 186 593-612 (1990)

16. Zaidenberg, M.G.: On exotic algebraic structures on affine spaces. St. Petersburg Math. J. 11 703-760 (2000) 